Voix et Images

volxetimages

\title{
Bibliographie de Yolande Villemaire
}

\section{Lucie Robert}

Volume 11, numéro 3 (33), printemps 1986

Yolande Villemaire

URI : https://id.erudit.org/iderudit/200580ar

DOI : https://doi.org/10.7202/200580ar

Aller au sommaire du numéro

Éditeur(s)

Université du Québec à Montréal

ISSN

0318-9201 (imprimé)

1705-933X (numérique)

Découvrir la revue

Citer ce document

Robert, L. (1986). Bibliographie de Yolande Villemaire. Voix et Images, 11(3),

455-462. https://doi.org/10.7202/200580ar d'utilisation que vous pouvez consulter en ligne.

https://apropos.erudit.org/fr/usagers/politique-dutilisation/ 


\title{
Bibliographie de Yolande Villemaire
}

\author{
par Lucie Robert, Université Laval
}

Plan de la bibliographie:

\section{OEUVRES}

I.I VOLUMES

I.2 OUVRAGES EN COLLABORATION

I.3 THĖSE

I.4 ARTICLES DE PÉRIODIQUES

II ÉTUDES

\section{II.1 GÉNÉRALITÉS}

\section{II.2 SUR LES CEUVRES}

II.21 Adrénaline; II.22 Ange Amazone; II.23 Belles de nuit; II.24 Les Coïncidences terrestres; II.25 La Constellation du Cygne; II.26 Du côté hiéroglyphe de ce qu'on appelle le réel; II.27 Jeunes Femmes rouges toujours plus belles; II.28 Machine-t-elle; II.29 Meurtres à blanc; II.30 Quartz et mica; II.31 Que du stage blood; II.32 La Vie en prose; II.33 Divers.

\section{OEUVRES}

\section{I.1 VOLUMES}

Meurtres à blanc. Roman. Montréal. Guérin. 1974. 164 p. (coll. le Cadavre exquis).

Machine-t-elle. Poésie, Montréal, les Herbes rouges, 22 (juillet 1974), n.p. Un extrait parut dans Action poétique, 93 (1983), p. 49-52.

Que du stage blood, Montréal, les Éditions Cul Q, 1977, 44 p. (coll. Exit, 2).

Terre de mue. Montréal, les Éditions Cul Q. 1978, n.p. (coll. Mium. Mium, 20). Des extraits parurent dans Hobo/Québec, 34 (septembre-octobre 1977). p. 4-5, et dans Action poétique, 93 (1983), p. 49-52.

Elle dit qu'elle dit que... Poème-affiche, distribué dans les autobus de la CTCUM. Montréal. Poésie en mouvement, 1980.

"La Version d'amour", dans Nicole Brossard. French Kiss. Étreinte/exploration. Roman, Montréal, Quinze. 1980. 157 p. (coll. Présence). v. p. 7-10.

La Vie en prose, Montréal, les Herbes rouges, 1980, 261 p. (coll. Lecture en vélocipède, 25); 1984. 372 p. (coll. Typo, 2); Des extraits parurent dans Hobo/Québec, $36-37$ (janvier-mars 1979), p 6-7; $41-42$ (été 1980), p. 56-57. Prix des jeunes écrivains du Journal de Montréal, 1980. 


\section{VOIX \& IMAGES/33, printemps 1986}

Ange Amazone. Roman, Montréal, les Herbes rouges, 1982, 100 p. (coll. Lecture en vélocipède). Adrénaline. Poésie et prose 1973-1982. graphisme et photographie de Michel Lemieux, SaintLambert, Éditions du Noroît, 1982, 172 p. "TilT" parut d'abord dans Cul-Q. 1 (automne 1973), p. 10-11; “Snoopy détective" la Barre du jour. 44 (printemps 1974), p. 23-35; "Tic Tac soluble dans le fortran", Cul-Q. 4-5 (été-automne 1974), p. 63-68; "Adrénalines" suivi de "Qui capote, vu de face», Cul-Q, 6-7 (1975), n.p.; "Circa 1975», Cul-Q, 6-7 (printemps 1975), n. p.; "Pour une parthénogénèse de la parole 'hystérique" ("Matrice vierge»), la Barre du jour. 50 (hiver 1975), p. 37-44; "Mon cœur battait comme un bolo", la Barre du jour. 56-57 (mai-a oût 1977), p. 116-136; "My heart beats like a bolo", traduction de Barbara Godard, Room of one's own", IV, 2 (1978); "la Grande Ourse - configuration du désir et de la peur. schéma de la possession". la Nouvelle Barre du jour. 64 (mars 1978), p. 57-96; «la Pyramide des j. The Pyramids of iiis", traduction de Ray Chamberlain, textes français et anglais en regard, dans Nicole Brossard (éditrice), les Stratégies du réel. The Story so far 6 , Montreal, «la Nouvelle Barre du jour", et Toronto, The Coach House Press, 1979, 341 p. v. p. 35-55; “ Extrait du livre de bord de l'expédition Dalst/Swannson sur Polaris. Les Dernières volontés de Will H. Dalst", la Nouvelle Barre du jour. 90-91 (mai 1980). p. 173-190; "Pareil comme dans 'la Nuit des temps'”, la Nouvelle Barre du jour. $92-93$ (juin 1980), p. 247-251; "Estelle toute la nuit en elle», la Nouvelle Barre du jour. 100-101 (mars 1981), p. 144-148; " les Anges incognito", la Nouvelle Barre du jour, 102 (avril 1981), p. 33-42; "Une question de vitesse. Tempête de neige. Ma sceur la peur», Arcade. 1 (printemps 1982). p. 42-44; «Miguel Ramon". Liberté, XXIV. 2. no 142 (juillet-août 1982), p. 42-52.

Du côté hiéroglyphe de ce qu'on appelle le réel, suivi de Devant le temple de Louxor le 31 juillet 1980. Montréal, les Herbes rouges, 102-103 (avril-mai 1982). 73 p. Des extraits parurent dans Hobo-Québec, 38-39 (automne 1979), p. 44; sous le titre "Pan, dieu de la Terreur panique, viens à mon secours!",Hobo-Québec, 40 (hiver 1979-1980), p. 13; "Tu me demandes ce que c'est l'Égypte", Hobo-Québec, 43 (hiver 1980-1981), p. 51.

Belles de nuit. Pièces radiophoniques, Montréal, les Herbes rouges, 1983, 183 p. (coll. Lecture en vélocipède). "Les Égoûts de New York": dramatique de 30 minutes, diffusée dans le cadre de l'émission "Premières" à Radio-Canada MF, le 11 mai 1979, dans une réalisation de JeanPierre Saulnier. "Belles de nuit»: dramatique de 60 minutes, diffusée dans le cadre de "Premières" à Radio-Canada MF, le 27 mai 1983, dans une réalisation de Jean-Pierre Saulnier; prix du concours des œuvres radiophoniques de Radio-Canada, 1980

Les Coincidences terrestres. Montréal, les Éditions de la Pleine Lune, 1983. 33 p. (coll. Rrose Sélavy).

Jeunes Femmes rouges toujours plus belles. Montréal, Lèvres urbaines, 8 ( $3^{\mathrm{e}}$ trimestre 1984$)$, p. 24. Un extrait parut dans la Nouvelle Barre du jour. 75 (février 1979), p. 48-60.

La Constellation du Cygne. Montréal, les Éditions de la Pleine Lune, 1985, 179 p. (coll. Rrose Sélavy). Un extrait parut sous le titre "Celia Rosenberg" la Nouvelle Barre du jour, 122-123 (février 1983), p. 205-209. Grand prix littéraire du Journal de Montréal, section roman, 1985.

Quartz et mica, Trois-Rivières, Écrits des Forges, et Pantin (France), le Castor astral, 1985. 54 p. (coll. Co-édition, no 2).

\section{2 OUVRAGES EN COLLABORATION}

Au fond des yeux, 25 Québécoises qui écrivent, album photographique de Kero, Montréal. Nouvelle Optique. $1981,109 \mathrm{p}$.

Anthologie 80. Bilan et perspectives de la poésie franco-belge-québécoise (auteurs nés après 1930, Talence/Herstal, le Castor astral/l'Atelier de l'agneau, 1981, 559 p. (p. 411).

Dix nouvelles humoristiques par dix auteurs québécois, collectif sous la direction d'André Carpentier, Montréal, Quinze, 1984. 221 p. "Ça devient clair en 1984", p. 199-220.

Rrose Sélavy à Paris, le 28 octobre 194l, Montréal, les Éditions de la Pleine Lune, 1984, 117 p. (coll. Rrose Sélavy).

Vouloir la fiction. La modernité, $2^{\epsilon}$ colloque bissextile, 29 février 1984. la Nouvelle Barre du jour, no 141 (septembre 1984), p. 8-76.

Rrose Sélavy, Spirale d'écrivantes, Arcade, 6 (automne 1984). 


\section{I.3 THËSE}

"Éléments d'une morphologie de l'œuvre dramatique de Michel Tremblay ( $A$ toi pour toujours, ta Marie-Lou)". Thèse de maîtrise ès arts, Montréal, Université du Québec à Montréal, 1973. iii, 191 f. Appendices A-D.

\subsection{ARTICLES DE PERIODIQUES}

“Baloune parlante", les Herbes rouges, 9 (juin 1973), n. p.

«Showtime. Hosanna ou la Mort du diable», Hobo-Québec, I. 8 (juin 1973), p. 22.

«Showtime. Yvon Dupuis, superstar», Hobo-Québec, 1, 9-11 (octobre-novembre 1973), p. 56-57.

"Showtime. Pourquoi attendre la postérité? Devenez une légende maintenant», Hobo-Québec; 12-13 (décembre 1973), p. 32-33.

"Showtime. UQAM. Quat'Sous. TNM ", Hobo/Québec, 14-15 (janvier 1974), p. 37-38.

«Showtime», Hobo/Québec, 16-17 (février 1974), p. 33-34, 38. Sur "Un prince, mon jour viendra".

«La N.C.T. au fil des saisons", les Cahiers de la Nouvelle Compagnie théâtrate. VIII, 3 (mars 1974). p. 13-16.

"La Scène érotomane", Cul-Q, 2-3 (hiver-printemps 1974), p. 7-20.

"Le Théâtre d'la Shop: un théâtre militant", Hobo/Québec, 18 (avril-mai 1974), p. 6-10.

"Showtime. Collage Montreal/New York", Hobo/Québec, 19 (septembre-octobre 1974), p. 24.

"Créations et Adaptations de Michel Tremblay", les Cahiers de la Nouvelle Compagnie théâtrale, IV, 1 (octobre 1974), p. 6-13.

"Les Pouvoirs de la parole". les Cahiers de la Nouvelle Compagnie théâtrale, IX, I (octobre 1974), p. 20-21.

"Showtime. L'Atem/théâtre", Hobo/Québec, 21 (janvier-février 1975), p. 16-17.

"Showtime. La Bebitte à Roche (Rushes)». Hobo/Québec, 23-24 (mai-août 1975), p. 6-8, 17-19. “Showtime. Le Théâtre des Cuisines», Hobo/Québec, 25 (septembre-décembre 1975), p. 6-8.

"Un homme de théâtre", les Cahiers de la Nouvelle Compagnie théâtrale, X. 1 (octobre 1975), p. 3-10, 19-20. Sur Gratien Gélinas.

“Vient de paraitre. Cé tellement 'cute' des enfants», le Jour, IIl. 6 (5 mars 1976), p. 28.

"Autour de la Nef des sorcières", Jeu, 2 (printemps 1976), p. 16-21.

"Spectacles. Soirée d'actions collectives». Jeu. 2 (printemps 1976), p. 93-95.

“'Jeudi soir en pleine face’ de Michel Beaulieu". Jeu, 2 (printemps 1976), p. 98-99.

"Showtime", Hobo/Québec, 29-30 (mai-a out 1976), p. 25-26. Sur "Un pays dont la devise est je m'oublie", "la Nef des sorcières» et le Groupe de la Veillée.

"Les Héros de mon enfance», Jeu, 3 (été-automne 1976), p. 74-77.

"Showtime», Hobo/Québec, 31 (septembre-décembre 1976). p. 25-27. Sur le groupe de la Veillée. "Cartographie d’un événement ", Jeu, 4 (hiver 1977), p. 105-107.

«Jeu de cartes (fragments)", Jeu, 4 (hiver 1977), p. 14-15.

"Showtime», Hobo/Québec, 32 (janvier-mars 1977), p. 6-7. Sur Ines Pérée et Inat Tendu.

"Showtime», Jeu, 5 (printemps 1977), p. 65-70. Sur la reprise de «Un prince, mon jour viendra». "Showtime", Hobo/Québec, 33 (avril-juin 1977), p. 3-4. Non vidi.

«L'Amèr ou le Chapitre effrité», la Nouvelle Barre du jour. 59 (octobre 1977), p. 84-87.

"La nuit expérimentale", Hobo/Québec, 35 (1978), p. 6-7.

«e», Jeu, 7 (hiver 1978), p. 99-100.

«La Chienne de l'hôtel Tropicana, de Josée Yvon", Mainmise/ Rézo, 73 (janvier 1978), p. 40. Non vidi.

"ll était une fois dans l'est: l'empire des mots", Jeu, 8 (printemps 1978), p. 61-75.

"L'Heure du break", Sorcières. 14 (septembre 1978), p. 37-39.

"L'Aleph-silence", Atelier de production littéraire de la Mauricie, 8 (1979), p. 89-90.

"Dire oui à soie: Alice a la peau rouge et ne se met pas de fond de teint ». Spirale, 1 (septembre 1979), p. 13. 


\section{VOIX \& IMAGES/33, printemps 1986}

"Tout seul comme deux": Une démarche peu courante», Spirale. 3 (novembre 1979), p. 3. "Yvelle Swannson: Pour l'imaginaire", le Devoir, LXX, 281 (1 ${ }^{\text {er }}$ décembre 1979), p. 43. «Être une héroïne». Spirale. 5 (janvier 1980), p. 10.

"Ah! comme la neige a neigé...", le Temps fou (supp. la Vie en rose), 9 (mars-mai 1980), p. 22-23.

"Plurielles. Performantes", Jeu, 16 ( $3^{\mathrm{e}}$ trimestre 1980), p. 175-177.

"Astéroïde 823 (histoire d'écrire)". la Nouvelle Barre du jour, 97 (décembre 1980), p. 38-79.

"L'Équinoxe de printemps 1981",Hobo/Québec, 44-45 (printemps-été 1981), n.p.

"Du côté hiéroglyphe de ce qu'on appelle le réel". Communication à la rencontre internationale des écrivains québécois, Liberté, XXIII, 4, no 136 (juillet-août 1981), p. 62-68. Suivi d'un débat, p. 71-83.

"Écrire en couleurs", le Devoir, LXXII, 222 (28 novembre 1981), p. 24. Reproduit dans les Deux rives, I (printemps-été 1984), p. 22-23.

"Portes ouvertes II - Quand Dieu était une femme", Lettres québécoises, 26 (été 1982), p. 80-81.

“La Machine à imaginer", Arcade, 2 (automne 1982), p. 5-13.

"La Tour de Babel et le Serpent vert de Goethe" Liberté, XXIV, 5, no 143 (octobre 1982), p. 77-106.

"Pourquoi j'écris", Québec français, 47 (octobre 1982), p. 33.

"Vénus est une jeune femme rouge toujours plus belle", la Nouvelle Barre du Jour, 118-119 (novembre 1982), p. 45-61.

«Régionalisme. Internationalisme. Un rapport de forces (Débat), Possibles. VII, 1 ( $4^{e}$ trimestre 1982), p. 53-71.

«Lecture co(s)mique en couleurs de Speak White de Michèle Lalonde", Possibles, VII, 1 (4 trimestre 1982), p. 161-163.

"Sarpa Rajni et le râckoeur", Arcade, 3 (hiver 1983), p. 61-63.

"Blues d'amour de nuit», Liberté, XXV, 1, no 145 (février 1983), p. 82-83.

"Rrose Sélavy", la Vie en rose, 11 (mai 1983), p. 57.

"Fiction. Dana Khan", la Vie en rose, 12 (juillet 1983), p. 48-52.

"Choisir", Estuaire, 32-33 (été-automne 1984), p. 137-138.

"Dessiner l'hologramme du futur", le Devoir, LXXV, 268 (17 novembre 1984), p. I, XIX.

"Signe de piste en blanc». Atelier de production littéraire des Forges. 19-20 ( $1{ }^{\mathrm{er}}$ trimestre 1985). p. 17-21.

"Mémoire-mémoires", Cahiers des arts visuels au Québec, VII, 25 (printemps 1985). Non vidi. "Rrose Sélavy, New York 1921". Between C and D (Lower East Side Post-Literate Fiction Magazine) (New York), Il, 2 (Fall 1985), n.p.

"This is the Space of Miracles", Lèvres urbaines, 11 (septembre 1985), p. 19.

\section{II ÉTUDES}

II.I GÉNÉRALITÉS

Anonyme, "Voici ce que quelques écrivains... écrivent ... au sujet de l'écriture et de la lecture". l'Écrilu, 1, 2 (septembre 1981), p. 11.

-, "Montréalais dans les studios du Québec à New York et à Paris», le Devoir, LXXV, 212 (12 septembre 1984 ), p. 7.

BEAUSOLEIL, Claude, "la Poésie en revues depuis 10 ans», la Petite Revue de philosophie, IV, I (automne 1982), p. 116.

BLOUIN, Louise et Bernard POZIER, "Entretien avec Yolande Villemaire», Atelier de production littéraire des Forges, 19-20 ( ${ }^{\text {er }}$ trimestre 1985), p. 6-16.

CHEVRIER, Chantal, «Écrire: une bande dessinée co(s)mique», Virus. VI, 8 (automne 1983), p. 20-21. Entrevue. 
DU PRÉ, Louise, «Poésie et Féminisme. De la chair à la langue", la Vie en rose, 11 (mai 1983), p. 54-55.

ESCOMEL, Gloria, "Littérature. Pourquoi écrire aujourd'hui", la Vie en rose, 22 (décembre 1984-janvier 1985), p. 54-55.

HAMEL, Réginald, John HARE et Paul WYCZYNSKI, Dictionnaire pratique des auteurs québécois. Montréal, Fides, 1976, p. 685-686.

MAISONNEUVE, Lise, "Entrevue avec Yolande Villemaire", Prélexte, II, 2 (1 ${ }^{\mathrm{cr}}$ trimestre 1981), p. 9-17.

PETROWSKI, Nathalie, "Villemaire-on-the-Hudson», le Devoir. LXXVI, 85 (13 avril 1985), p. 21.

ROYER, Jean, «la Littérature québécoise d'Amérique. Yolande Villemaire», les Deux rives, I (printemps-été 1984), p. 19-21. Entrevue.

UNION DES ÉCRIVAINS QUÉBÉCOIS, Dictionnaire des écrivains québécois contemporains. recherche et rédaction Yves Légaré, Montréal, Québec/Amérique, 1983. p. 391.

\section{II.2 SUR LES CEUVRES}

\section{II.21 Adrénaline}

BAYARD, Caroline, "Letters in Canada. Poésie», University of Toronto Quarterly, LIl, 4 (Summer 1983), p. 359-360.

—. "Marie-José Thériault, Yolande Villemaire, Jovette Marchessault", Lettres québécoises, 29 (printemps 1983), p. 42-45. v. p. 44.

BEAUSOLEIL, Claude, les Livres parlent, Trois-Rivières, Écrits des Forges. 1984, p. 219-225.

BOUCHARD, Christian, “Estuaire a reçu», Estuaire, 27 (printemps 1983), p. 89.

CHAM BERLAND, Roger, «Poésie», Québec français, 48 (décembre 1982). p. 6.

CORRIVEAU, Hugues, «Poésie québécoise. Pulsion urgente», Spirale, 33 (avril 1983), p. 4.

MÉLANÇON," Robert, "Du même auteur», Liberté, XXV, 2, no 146 (avril 1983), p. 117-119.

NEPVEU, Pierre, «Adrénaline», Livres et Auteurs québécois. 1982, p. 146-148.

\section{II.22 Ange Amazone}

CHAMBERLAND, Roger, "Poésie», Québec français, 48 (décembre 1982), p. 6.

DAVID, Carole, "Roman québécois. Le Corps blessé de Wonder Woman», Spirale, 28 (octobre 1982), p. 6.

FERRETTI, Andrée, "Lettre à l'Ange Amazone", le Devoir, LXXIII, 251 (30 octobre 1982), p. 22. Reproduit dans les Deux rives, 1 (printemps-été 1984), p. 24.

GILBERT, Bernard, «Littérature québécoise. Commentaires (...) Ange Amazone», Nuit blanche. 7 (automne 1982), p. 10.

LAPIERRE, René, «Yolande Villemaire. À l'épreuve du langage et du monde», le Devoir, LXXIII, 222 (25 septembre 1982), p. 19.

LEBLANC, Gérard, "Traduire Ange Amazone", Atelier de production littéraire des Forges, 19-20 ( $1^{\text {ci }}$ trimestre 1985). p. 31.

LÉPINE, Stéphane, "Villemaire (Yolande). Ange Amazone», Nos livres, XV, 5661 (mars 1984), p. 34-35.

LETARTE, Genesse, "Ange Amazone», le Temps fou, 25 (février 1983), p. 60.

MÁLENFANT, Paul Chanel, "Du côté hiéroglvphe de ce qu'on appelle le réel - AngeAmazone», Livres et Auteurs québécois, 1982, p. 83-84.

MARTIN, Raymond, "Ange Amazone", Moebius, 16 (hiver 1983), p. 68.

MÉLANÇON, Robert, "Du même auteur», Liberlé, XXV, 2, no 146 (avril 1983), p. 117-119.

SALESSE. Michèle, "Porte ouverte I. Yolande Villemaire et le dieu Ptah", Lettres québécoises, 27 (automne 1982), p. $87-88$

STANTON. Julic, "Culture (...) Ange Amazone", la Gazente des femmes, IV, 6 (janvier-février 1983), p. 4.

VISWANATHAN, Jacqueline, "Dire le moi», Canadian Literature, 102 (Autumn 1984), p. 129-130. 


\section{II.23 Belles de nuit}

BEAUSOLEIL. Claude, "le Langage de ces Belles de nuit", Atelier de production littéraire des Forges, 19-20 ( ( $^{\text {er }}$ trimestre 1985), p. 22-24.

GRISÉ, Yolande, "Théâtre en ondes. Théâtre en secondes", Spirale, 45 (septembre 1984), p. 6.

LAURIN, Michel, "Villemaire (Yolande). Belles de nuit», Nos livres, XV, 5706 (avril 1984). p. 29-30.

SALESSE, Michèle, "Belles de nuit de Yolande Villemaire», Lettres québécoises, 34 (été 1984), p. 92.

\section{II.24 Les Coüncidences terrestres}

ALONZO, Anne-Marie, "Alexandrie en 1930", la Vie en rose, 15 (janvier-février 1983), p. 63.

BEAUSOLEIL. Claude, "Mots d'ailleurs et de la Terre", le Devoir. LXXV, 41 ( 18 février 1984), p. 21 .

—, "Villemaire (Yolande). Les Cö̈ncidences terrestres», Nos livres, XV, 5759 (mai 1984), p. 36.

—, les Livres parlent, Trois-Rivières, Écrits des Forges, p. 219-225.

CHEVRIER, Chantal, "Écrire: une bande dessinée co(s)mique», Virus, VI, 8 (octobre 1983), p. 20-21. Entrevue.

GAUDET, Gérald, "Puisqu’écrire est l'obscur du désir», Atelier de production littéraire des Forges, 19-20 (1 $1^{\text {er }}$ trimestre 1985), p. 25-27.

GIGUERE, Suzanne et Marie-Claude TRÉPANIER, "Yolande Villemaire. Interview», Lettres québécoises, 40 (hiver 1985-1986), p. 50-52.

\section{II.25 La Constellation du Cygne}

BOIVIN, Jean-Roch, “Informer. Lire en vacances», Décormag, 143 (juillet-août 1985), p. 18.

CHAMBERLAND, Roger, "Romans. La Constellation du Cygne», Québec français, 59 (octobre 1985), p. 8.

CHASSAY, Jean-François, «Roman québécois. Une histoire sans l'Histoire», Spirale, 52 (mai 1985). p. 5.

FOURNIER, Danielle. "Yolande Villemaire. La Constellation du Cygne. Annie Leclerc. Hommes et Fermmes», Moebius, 26 (automne 1985), p. 120-122.

GIGUĖRE, Suzanne et Marie-Claude TRÉPANIER, "Yolande Villemaire. Interview", Lettres québécoises, 40 (hiver 1985-1986), p. 50-52.

HÉBERT, Frạç̧ois. "Une nouvelle piété? Lettres québécoises", le Devoir, LXXVI, 80 (6 avril 1985), p. 21.

MARCOTTE, Gilles, "Lectures. Trois conteurs de charme...", Actualité, X, 6 (juin 1985). p. 133.

MARTEL. Réginald, «Yolande Villemaire. Jouissance et mort de Célia Rosenberg», la Presse, CI, 146 (16 mars 1985), p. E-3.

PELLETIER, Francine, «Livres. Guerre et passion», la Vie en rose, 26 (mai 1985), p. 54.

POULIN, Gabrielle, "Une mécanique solennelle. La Constellation du cygne de Yolande Villemaire", Lettres québécoises, 39 (automne 1985), p. 21-23.

TURCOTTE, Suzy, «Littérature québécoise. Commentaires (...) La Constellation du Cygne», Nuit blanche, 19 (juin-juillet 1985), p. 10.

TURGEON, Carole, "Villemaire (Yolande). La Constellation du Cvgne», Nos livres, XVI, 6259 (juin-juillet 1985), p. 44-45.

\section{II.26 Du côté hiéroglyphe de ce qu'on appelle le réel}

BEAUSOLEIL, Claude, «le Prétexte égyptien», la Nouvelle Barre du jour. 129 (septembre 1983), p. 101-110. v. p. 105-107.

-. les Livres parlent, Trois-Rivières, Écrits des Forges, 1984, p 219-225.

BROCHU, André, «Des femmes et des mots», Voix et images, VIII, 3 (printemps 1983). p. 503-510.

CHAMBERLAND, Paul, préface du livre, p. 3-8.

CHAMBERLAND, Roger, "Poésie», Québec français, 48 (décembre 1982), p. 6. 
LAPIERRE, René, "Yolande Villemaire. À l'épreuve du langage et du monde", le Devoir, LXXIII, 222 (25 septembre 1982), p. 19.

MALENFANT, Paul Chanel, "Du côté hiéroglyphe de ce qu'on appelle le réel - Ange Amazone", Livres et Auteurs québécois, 1982, p. 83-84.

MÉLANÇON, Robert, "Du même auteur», Liberté, XXV, 2, no 146 (avril 1983), p. 117-119.

SALESSE, Michèle, "Poste ouverte I. Yolande Villemaire et le dieu Ptah", Lettres québécoises, 27 (automne 1982), p. 87-88.

\section{II.27 Jeunes Femmes rouges toujours plus belles}

LEPINE, Stéphane, "Villemaire (Yolande). Jeunes Femmes rouges toujours plus belles", Nos livres, XV, 5954 (novembre 1984), p. 39-40.

POZIER, Bernard, "le Temps des passions", Atelier de production littéraire des Forges, 19-20 ( $1^{\text {er }}$ trimestre 1985). p. 28-29.

ROYER, Jean, "Chronique pour non-liseurs de poésie. De l'érotisme avant toute chose", le Devoir, LXXVI, 57 (9 mars 1985), p. 24.

TOUPIN, Gilles, "Poésie d'ici. Villemaire et Alonzo", la Presse, CI, 57 (15 décembre 1984), p. E-4.

YERGEAU, Robert, "Poésie. L'Amour la poésie», Lettres québécoises, 38 (été 1985), p. 30-32. v. p. 31 .

\section{II.28 Machine-t-elle}

GIGUĖRE, Richard, «les Herbes rouges: une grande 'petite revue'", Livres et Auteurs québécois. 1975, p. 118-121. v. p. 119-120.

\section{II.29 Meurtres à blanc}

PLANTE, Raymond, “Des problèmes à plusieurs inconnus», la Presse, XC, 160 (6 juillet 1974), p. E-2.

\section{II.30 Quartz et mica}

ALONZO, Anne-Marie, "De l'or en spray», la Vie en Rose, 33 (février 1986), p. 56.

POPOVIC. Paul. «Poésie québécoise. Interstices, intermittences et non-advenu», Spirale, 58 (février 1986), p. 10.

ROYER. Jean, "Chronique pour non-liseurs de poésie. Car la vie est un roman", le Devoir, LXXVI, 219 (2l septembre 1985), p. 26.

\section{II.31 Que du stage blood}

RENAUD, Jacques, "Poètes québécois en quête de l'amour des instincts et de la source", le Devoir, LXIX, 185 (13 août 1977), p. 12.

\section{II.32 La Vie en prose}

(Anonyme), «les Prix littéraires du Journal de Montréal», Lettres québécoises, 21 (printemps 1981), p. 11.

ALMÉRAS, Diane, "la Vie en prose et en couleurs», Relations, XLI, 467 (février 1981), p. 60-61.

BAYARD, Caroline, "Letters in Canada. Poésie", University of Toronto Quarterly, L, 4 (Summer 1981), p. 41-55. v. p. 44.

BEAUSOLEIL, Claude, "Écritures de ville", la Nouvelle Barre du jour, 99 (février 1981), p. 75-78. v. p. 76-77.

BOURQUE, Paul-André, "Letters in Canada. Romans", University of Toronto Quarterly, L. 4 (Summer 1981), p. 20-27. v. p. 23-24.

CHAMBERLAND, Roger, «la Vie en prose», Livres et Auteurs québécois. 1980, p. 77-78.

-. "Romans", Québec français, 42 (mai 1981), p. 15.

CHASSAY, Jean-François, "la Stratégie du désordre: une lecture de textes montréalais", Études françaises, XIX, 3 (hiver 1983-84), p. 93-103.

-, «Structures urbaines. Structures textuelles: la ville chez Réjean Ducharme, David Fennario, Yolande Villemaire". Thèse de maîtrise ès arts, Montréal, Université de Montréal, 1984, $154 \mathrm{f}$. 


\section{VOIX \& IMAGES/33, printemps 1986}

DUMONT, Monique, «la Vie en prose», la Vie en rose, II, 1 (mars-mai 1981), p. 46.

LAMY, Suzanne, Quand je lis je m'invente. Essai, Montréal, l'Hexagone, 1984, 111 p. passim.

LAPIERRE, René "Littérature québécoise. Rose c'est la vie", Liberté, XXIII, 1 no 133 (janvierfévrier 1981), p. 83-85.

LARUE, Monique, "le Texte derviche», Spirale, 13 (novembre 1980), p. 4.

MARCOTTE, Gilles, «Lectures. Un suspense et une vie en rose», Actualité, VI, 2 (février 1981), p. 71-72.

MARTEL, Réginald, "Villemaire et Lanctôt. Du flou au réel", la Presse, XCVII, 14 ( 17 janvier 1981), p. C-3.

OUELLETTE-MICHALSKA, Madeleine, "la Mort de la dame en rose", le Devoir, LXXI, 263 ( 15 novembre 1980), p. 23.

POZIER, Bernard, "Lauréate du prix du Journal de Montréal. La Vie en prose de Yolande Villemaire: un roman-journal», le Nouvelliste, LXI, 27 (29 novembre 1980), p. 23. Reproduit sous le titre "la Vie en prose à revisiter", Atelier de production littéraire des Forges, 19-20 (1 ${ }^{\text {er }}$ trimestre 1985), p. 32-33.

\section{II.33 Divers}

DAOUST, Jean-Paul. «Rrose Sélavy», Atelier de production hittéraire des Forges, 19-20 (1 $1^{\text {er }}$ trimestre 1985), p. 30.

DUPRÉ. Louise. «Culture. Le féminisme de Rrose Sélavy», la Vie en rose, 17 (mai 1984), p. 56-57. Entrevue.

ROYER, Jean, “Jovette Marchessault: 'Il faut se rendre visible...': célébration des femmes au TNM », le Devoir. LXX. 52 (3 mars 1979), p. 19. Sur le spectacle Célébrations. 